\title{
ESTABELECIMENTO DE CONDIÇÕES DE LUZ E TEMPERATURA PARA GERMINAÇÃODE SEMENTES DE AMENDOIM FORRAGEIRO ${ }^{1}$
}

\author{
ANA LAURA PEREIRA AMATO², FERNANDA COSTA MAIA ${ }^{3}$, MANOEL DE SOUZA MAIA, ${ }^{4}$ \\ LEANDRO SEBASTIÃO CAETANO ${ }^{5}$, SILVIA BRISTOTT SIMIONI ${ }^{5}$, LEANDRO DE CONTO $^{5}$, \\ ROBERTO DE MOURA BONINI FILHO ${ }^{6}$
}

\begin{abstract}
RESUMO - O objetivo do presente trabalho foi comparar diferentes condições de luz e temperatura para o estabelecimento do teste de germinação de sementes de amendoim forrageiro (Arachis pintoi Krapov. \& W.C. Gregory), visando uma primeira aproximação à padronização do teste. Os testes de germinação foram conduzidos com 200 sementes (quatro repetições de 50 sementes), utilizando-se como substrato rolo de papel toalha. $\mathrm{O}$ experimento foi conduzido em duas etapas. Na primeira, as temperaturas utilizadas foram $25^{\circ} \mathrm{C}, 30^{\circ} \mathrm{C}, 35^{\circ} \mathrm{C}, 25^{\circ}-30^{\circ} \mathrm{C}, 25^{\circ}-35^{\circ} \mathrm{C}$ e $30^{\circ}-35^{\circ} \mathrm{C}$, com e sem luz. Na segunda, as temperaturas alternadas de $25^{\circ}-30^{\circ} \mathrm{C}$ e $25^{\circ}-35^{\circ} \mathrm{C}$ foram comparadas com a recomendada para o amendoim comum (Arachis hypogaea L.), $20^{\circ}-30^{\circ} \mathrm{C}$ com luz. Foram utilizadas três repetições, em delineamento completamente casualizado. Verificou-se que a utilização de temperaturas alternadas promove uma diminuição significativa no número de sementes duras devido à remoção da dormência das sementes. Entre os tratamentos com temperatura constante, a de $30^{\circ} \mathrm{C}$ com luz promove o maior número de plântulas normais. As temperaturas alternadas de $20^{\circ}-30^{\circ} \mathrm{C} \mathrm{e} 25^{\circ}-30^{\circ} \mathrm{C}$, com luz, são as mais recomendadas para a condução do teste de germinação. Através da contagem diária do número de plântulas normais, recomenda-se a realização da primeira contagem aos 8 dias e o encerramento do teste de germinação aos 14 dias.
\end{abstract}

Termos para indexação: Arachis pintoi, forrageiras, teste de germinação, dormência.

\section{DETERMING LIGHT AND TEMPERATURE CONDITIONS FOR THE STANDARD GERMINATION TEST OF BRAZILIAN PEANUT}

\begin{abstract}
The objective of this study was to compare different light and temperature regimes to establish conditions for the standard germination test of Brazilian peanut (Arachis pintoi Krapov. \& W.C. Gregory). The germination tests were conducted with 200 seeds $(4 \times 50)$, using rolled paper towel. The experiment consisted of two stages. In the first, the temperatures used were $25^{\circ} \mathrm{C}, 30^{\circ} \mathrm{C}, 35^{\circ} \mathrm{C}, 25^{\circ}-30^{\circ} \mathrm{C}, 25^{\circ}-35^{\circ} \mathrm{C}$ and $30^{\circ}-35^{\circ} \mathrm{C}$, with and without light. In the second, the alternating temperatures of $25^{\circ}-30^{\circ} \mathrm{C}$ and $25^{\circ}-35^{\circ} \mathrm{C}$ with light were compared with the recommended $\left(20^{\circ}-30^{\circ} \mathrm{C}\right.$ with light) for peanut (Arachis hypogaea L.). Three statistical repetitions were used, in a completely randomized design. It was verified that the use of alternating temperatures promoted a significant decrease in the number of hard seeds because dormancy was overcome. Among the treatments with constant temperature, $30^{\circ} \mathrm{C}$ with light showed the highest number of normal seedlings. The temperatures of $20^{\circ}-30^{\circ} \mathrm{C}$ and $25^{\circ}-30^{\circ} \mathrm{C}$, with light, were the most recommended for the germination test. Daily countings of normal seedlings showed that the best day for the first counting was the $8^{\text {th }}$ and for the last one the $14^{\text {th }}$ day of the germination test.
\end{abstract}

Index terms: Arachis pintoi, forages, germination test, dormancy.

1 Submetido em: 09/10/2006Aceito para publicação em 11/04/2007.

2 Eng. Agr., Doutoranda PPG Ciência e Tecnologia de Sementes, Universidade Federal de Pelotas, anapereiraamato@ hotmail.com

3 Eng. Agr., Dr., Fundação Estadual de Pesquisa Agropecuária, Centro de Pesquisa da Região Sul, Rio Grande, RS, fecmaia@terra.com.br

4 Prof. Dr., Departamento de Fitotecnia, Faculdade de Agronomia Eliseu Maciel, Universidade Federal de Pelotas, Cx. Postal 354, Pelotas, RS, maiams@ufpel.edu.br

5 Acadêmicos do Curso de Graduação em Agronomia, Faculdade de Agronomia Eliseu Maciel, Universidade Federal de Pelotas.

6 Acadêmico do Curso de Graduação em Agronomia, Faculdade de Agronomia Eliseu Maciel, Universidade Federal de Pelotas, bolsista FAPERGS. 


\section{INTRODUÇÃO}

Existem poucas alternativas de leguminosas subtropicais utilizadas no Brasil e o amendoim forrageiro (Arachis pintoi Krapov. \& W.C. Gregory) constitui-se em uma espécie promissora tanto em produçãoe qualidade de matéria seca como na adaptação a diferentes ambientes. É uma espécie de utilização recente, com pouca informação disponível especialmente na área de produção e tecnologia de sementes.

O amendoim forrageiro é uma leguminosa estolonífera perene que apresenta grande abscissão de vagens na maturidade (Ferguson, 1995). É tolerante à sombra e relativamente tolerante a deficiências hídricas. Embora apresente rápido desenvolvimento da área foliar logo após a defoliação, apresenta melhor resposta para pastejo ou como cobertura do que para corte (Fisher e Cruz, 1995). No cerrado brasileiro, foram observadas produções de matéria seca do amendoim forrageiro entre 2 a 9 t/ha na estação das chuvas e 2 a 4 t/ha na estação da seca (Pizarro e Rincón, 1995). O valor nutritivo de sua forragem é maior que o da maioria das espécies de leguminosas tropicais atualmente comercializadas com um teor de proteína bruta da matéria seca de suas folhas entre $13 \%$ a $22 \%$, conforme resultados de Lascano (1995).

A planta apresenta um estabelecimento mais rápido por sementes do que por estolões, embora mais freqüentemente seja realizado em es em estruturas vegetativas em função da dificuldade da retirada das vagens do solo é difícil (Fisher e Cruz, 1995). A flor seca após a fecundação sem se desprender da planta, iniciando-se então, a formação de um pedúnculo denominado peg. Este cresce $2 \mathrm{~cm}$ para cima e se dobra até o solo, terminando por enterrar o ovário que está em sua extremidade (Ferguson et al., 1992). Cada vagem contém uma única semente, raramente duas. Nessa espécie de Arachis, os pegs são frágeis e comprimidos, o que dificulta a sua retirada do solo na ocasião da colheita, sendo necessário revolver e peneirar o solo para recuperálos. Com adequado manejo da cultura, a produção potencial de sementes pode alcançar $5 \mathrm{t} / \mathrm{ha}$, encontrando-se usualmente produções entre 1 a 2 t/ha dos 15 aos 18 meses após o estabelecimento (Ferguson, 1995).

Sementes recém colhidas apresentam altos níveis de dormência $(60 \% \%$ a $80 \%)$, podendo manter-se por um período de seis a oito meses. Esta dormência pode ser reduzida com tratamento térmico a $40^{\circ} \mathrm{C}$ por 14 dias. Alguns lotes reduzem a sua viabilidade logo após 10 meses de armazenamento (Ferguson, 1995).

Esta espécie, por ser de recente utilização, não apresenta ainda recomendações para a metodologia do teste de germinação nas Regras para Análise de Sementes - RAS (Brasil, 1992). A metodologia que vem sendo utilizada para o teste de germinação do amendoim forrageiro atualmente é a mesma empregada para o amendoim comum (Arachis hypogaea L.). O potencial forrageiro da espécie e sua adaptação a diferentes ambientes tem gerado uma demanda crescente no comércio de sementes, o que exige informações concretas para a condução do teste de germinação.

Para o amendoim comum, segundo as RAS (Brasil, 1992), podem ser utilizadas as temperaturas constantes de $25^{\circ} \mathrm{C}, 30^{\circ} \mathrm{C}$ ou as temperaturas alternadas de $20^{\circ}-30^{\circ} \mathrm{C}$. O substrato utilizado pode ser rolo de pano, rolo de papel ou entre areia. A primeira contagem realiza-se aos cinco dias e a contagem final aos 10 .

A luz é necessária para a germinação das sementes de várias espécies. Porém, a sensibilidade das sementes ao efeito da luz varia de acordo com a qualidade, a intensidade luminosa e o tempo de irradiação, bem como com o período e a temperatura durante o processo de embebição (Laboriou, 1983). Segundo Pons (2000) a resposta das sementes à luz varia muito entre espécies e entre lotes de sementes, podendo depender também de pré-tratamentos e das condições disponíveis durante o teste de germinação

A temperatura exerce influência no processo germinativo, tanto por agir sobre a velocidade de absorção de água, como sobre as reações bioquímicas que determinam todo o processo; afetando, portanto, não só o total de germinação, como também a velocidade (Bewley \& Black, 1994).

A temperatura ótima para a germinação varia de acordo com as espécies, sendo esta definida geneticamente e, também, em função das condições fisiológicas das sementes (Carvalho \& Nakagawa, 2000).

O objetivo do presente trabalho foi comparar diferentes condições de luz e temperatura para o estabelecimento do teste de germinação de sementes de amendoim forrageiro, visando uma primeira aproximação à padronização do teste. 


\section{MATERIAL E MÉTODOS}

O material estudado constou de sementes de amendoim forrageiro da cultivar Amarillo, procedentes da região sudeste do Brasil.

Foram conduzidos testes de germinação com 200 sementes (quatro repetições de 50 sementes), utilizandose como substrato rolo de papel toalha. $\mathrm{O}$ experimento foi realizado em duas etapas. $\mathrm{Na}$ primeira, as temperaturas utilizadas foram $25^{\circ} \mathrm{C}, 30^{\circ} \mathrm{C}, 35^{\circ} \mathrm{C}, 25^{\circ}-30^{\circ} \mathrm{C}, 25^{\circ}-35^{\circ} \mathrm{C}$ e $30^{\circ}$ $35^{\circ} \mathrm{C}$, com e sem luz, totalizando 12 tratamentos. $\mathrm{Na}$ segunda, os dois tratamentos que apresentaram melhores resultados na primeira etapa $\left(25^{\circ}-30^{\circ} \mathrm{C}\right.$ e $25^{\circ}-35^{\circ} \mathrm{C}$ com luz $)$, foram comparados com a temperatura de $20^{\circ}-30^{\circ} \mathrm{C}$, com luz, recomendada pelas RAS (Brasil, 1992) para $A$. hypogaea. A análise estatística seguiu a metodologia de um experimento fatorial em delineamento completamente casualizado com três repetições estatísticas.

O critério para avaliar a germinação consistiu na observação diária, para determinar o comprimento das plântulas que permitiu identificar as normais (aproximadamente 6 a $7 \mathrm{~cm}$ ). O último dia do teste foi aquele em que foi retirada a maioria das plântulas normais e anormais, permanecendo no substrato apenas as sementes duras e mortas.

Através do aplicativo WINSTAT, realizou-se análise de comparação de médias e teste de Duncan, com 5\% de probabilidade. A temperatura mais adequada foi estabelecida como sendo a que proporcionou a maior germinação no menor intervalo de tempo.

\section{RESULTADOS E DISCUSSÃO}

A resposta das sementes à luz é um dos fatores que controla o tempo de germinação e pode ser observada mais comumente em espécies de sementes pequenas (Pons, 2000). Nestas sementes, a luz serve mais como um sinal do que como um recurso à germinação. Somente a água, o oxigênio e a temperatura adequadas são pré-requisitos para o crescimento do embrião. Segundo Bewley e Black (1994), as respostas das sementes à luz são consideradas como sinais de controle da luz sobre a dormência, ao invés de um controle direto sobre a germinação.
$\mathrm{Na}$ primeira etapa do experimento, as temperaturas alternadas de $25^{\circ}-30^{\circ} \mathrm{C}$ com luz apresentaram a maior porcentagem de germinação, seguidas dos tratamentos $25^{\circ}$ $35^{\circ} \mathrm{C} \mathrm{e} 30^{\circ} \mathrm{C}$ ambos com luz e $25^{\circ}-35^{\circ} \mathrm{C}$ sem luz. Estes quatro tratamentos não diferiram significativamente entre si.

Não foi verificada diferença significativa entre a média de todos os tratamentos para o fator luz, com relação ao número de plântulas normais.

Quando comparados os tratamentos com a mesma temperatura entre os grupos com e sem luz, às temperaturas de $30^{\circ} \mathrm{C} \mathrm{e} 25^{\circ}-35^{\circ} \mathrm{C}$ apresentaram diferenças significativas. O tratamento de $30^{\circ} \mathrm{C}$ com luz apresentou maior percentual de plântulas normais e menor de sementes duras do que na ausência de luz, enquanto que o tratamento de $25^{\circ}-35^{\circ} \mathrm{C}$ com luz apresentou maior número de plântulas normais e menor número de plântulas anormais.

Os tratamentos sem luz apresentaram diferenças significativas entre si, onde as temperaturas alternadas de $25^{\circ}-30^{\circ} \mathrm{C}$ superaram o percentual de plântulas normais das outras temperaturas, não diferindo significativamente da temperatura constante de $25^{\circ} \mathrm{C}$. O tratamento a $35^{\circ}$ também diferenciou-se dos outros já que este inibiu completamente a germinação. De forma similar o tratamento de $35^{\circ} \mathrm{C}$ com luz apresentou uma porcentagem de germinação muito baixa (1\%) e a maioria das plântulas germinadas anormais (64\%), indicando ser esta a temperatura limite para a germinação da espécie. A temperatura de $30^{\circ} \mathrm{C}$, com luz, foi a que apresentou melhor percentual germinativo dentre as temperaturas constantes, não diferindo de $25^{\circ} \mathrm{C}$ com luz (Tabela 1).

Para os tratamentos sem luz, as temperaturas alternadas apresentaram menores percentagens de sementes duras comparado com as temperaturas constantes. Os tratamentos com luz apresentaram a mesma tendência, mas a superioridade das temperaturas alternadas em relação às constantes não foram significativas em todos os casos.

Desta forma, é possível afirmar que as temperaturas alternadas promoveram diferenças significativas no número de sementes duras ao final do teste, demonstrando uma tendência à remoção da dormência das sementes pelo uso desta alternância.

Revista Brasileira de Sementes, vol. 29, no 3, p. 61-66, 2007 
TABELA 1. Percentagens de plântulas normais e anormais, e de sementes mortas e duras de Arachis pintoi, submetidas a diferenres condições de luz e temperatura para o experimento 1.

\begin{tabular}{|c|c|c|c|c|}
\hline \multirow{2}{*}{ Tratamentos } & \multicolumn{2}{|c|}{ Plântulas } & \multicolumn{2}{|c|}{ Sementes } \\
\hline & Normais & Anormais & Mortas & Duras \\
\hline \multicolumn{5}{|l|}{ Com luz } \\
\hline $25^{\circ} \mathrm{C}$ & $53 \mathrm{bcd}$ & $14 \mathrm{f}$ & $8 \mathrm{~b}$ & $25 \mathrm{bcc}$ \\
\hline $30^{\circ} \mathrm{C}$ & 56 abcd & $18 \mathrm{def}$ & $8 \mathrm{ab}$ & $18 \mathrm{cde}$ \\
\hline $35^{\circ} \mathrm{C}$ & $1 \mathrm{f}$ & $64 \mathrm{a}$ & $7 \mathrm{~b}$ & $28 \mathrm{~b}$ \\
\hline $25-30^{\circ} \mathrm{C}$ & $67 \mathrm{a}$ & 16 ef & $7 \mathrm{~b}$ & $10 \mathrm{fg}$ \\
\hline $25-35^{\circ} \mathrm{C}$ & $62 \mathrm{ab}$ & 17 ef & $10 \mathrm{ab}$ & $11 \mathrm{efg}$ \\
\hline $30-35^{\circ} \mathrm{C}$ & 48 cde & 26 bcde & $8 \mathrm{~b}$ & $18 \mathrm{def}$ \\
\hline \multicolumn{5}{|l|}{ Sem luz } \\
\hline $25^{\circ} \mathrm{C}$ & $46 \mathrm{cde}$ & $21 \mathrm{cdef}$ & $7 \mathrm{~b}$ & $26 \mathrm{bcc}$ \\
\hline $30^{\circ} \mathrm{C}$ & $36 \mathrm{e}$ & $28 \mathrm{bcd}$ & $9 \mathrm{ab}$ & $27 \mathrm{~b}$ \\
\hline $35^{\circ} \mathrm{C}$ & $0 \mathrm{f}$ & 16 ef & $12 a b$ & $72 \mathrm{a}$ \\
\hline $25-30^{\circ} \mathrm{C}$ & $58 \mathrm{abc}$ & 22 cdef & $14 \mathrm{a}$ & $6 \mathrm{~g}$ \\
\hline $25-35^{\circ} \mathrm{C}$ & $43 \mathrm{de}$ & $31 \mathrm{bc}$ & $10 \mathrm{ab}$ & 16 ef \\
\hline $30-35^{\circ} \mathrm{C}$ & $43 \mathrm{de}$ & $34 \mathrm{~b}$ & $8 \mathrm{~b}$ & 15 ef \\
\hline
\end{tabular}

As médias dentro de cada coluna seguidas da mesma letra não diferem entre si pelo teste de Duncan, a 5\% de probabilidade.

A condição mais adequada para germinação de sementes de uma determinada espécie é aquela que permite alcançar o maior número de plântulas normais, associado a um menor número de plântulas anormais e sementes mortas e dormentes, no menor tempo. Na primeira etapa do experimento, o tratamento que apresentou tal condição foi $25^{\circ}-30^{\circ} \mathrm{C}$ com luz.

Em um grande número de espécies, a germinação das sementes não ocorre ou é severamente prejudicada em ambientes de temperatura constante (Probert, 2000). Em 85 espécies selecionadas dentro de 15 famílias diferentes, Steinbauer e Grigsby (1957) observaram que, mais de $80 \%$ apresentou maior percentual germinativo com temperaturas alternadas do que com as constantes.

Nos testes de laboratório, as temperaturas alternadas são usadas para simularem as flutuações que normalmente ocorrem na natureza. Sabe-se que sementes de amendoimforrageiro se apresentam em dormência primária até cerca de 6 a 8 meses após a colheita, condição esta que pode ser reduzida mediante a pré-secagem por 14 dias a $40^{\circ} \mathrm{C}$ (Ferguson, 1995).

Rocha (1975) afirma que o uso de temperaturas alternadas para sementes reconhecidamente dormentes de certas espécies, com mudanças bruscas de um nível para outro, promove alta porcentagem de germinação.
Em muitos casos, a sensibilidade das sementes a temperaturas alternadas pode ser influenciada por outros fatores ambientais, particularmente pela luz (Toole e Koch, 1977; Roberts e Benjamin, 1979; Totterdell e Roberts, 1980; Probert et al., 1986). Neste estudo, os dados obtidos de número de plântulas normais demonstram uma interação significativa para estes dois fatores, apesar de não demonstrar efeito significativo do fator luz isoladamente. Tanto o fator luz quanto o fator temperatura e a interação de ambos influenciaram o número de plântulas anormais e de sementes duras.

As temperaturas alternadas que apresentaram os maiores percentuais germinativos nesta primeira etapa foram comparadas numa segunda etapa de testes de germinação com a temperatura de $20-30^{\circ} \mathrm{C}$ com luz, recomendada pelas RAS (1992) para o amendoim comum (A. hypogaea). Conforme pode-se observar na Tabela 2, os testes de germinação realizados na segunda etapa, não mostraram diferença significativa entre as temperaturas de $20-30^{\circ} \mathrm{Ce} 25-$ $30^{\circ} \mathrm{C}$, que apresentaram percentuais germinativos de 73 e $66 \%$, respectivamente, indicando que para A. hypogaea e A. pintoi pode ser utilizada a mesma condição de luz e temperatura. A temperatura de $25-35^{\circ} \mathrm{C}$ apresentou um percentual germinativo significativamente menor $(62 \%)$ quando comparado à temperatura de $20-30^{\circ} \mathrm{C}$, mas não diferindo do percentual observado na temperatura de $25-30^{\circ} \mathrm{C}$. 
TABELA 2. Percentagens de plântulas normais e anormais e de sementes mortas e duras de $A$. pintoi nas temperaturas alternadas recomendadas para a mesma $\left(25^{\circ}-30^{\circ} \mathrm{C}\right.$ e $\left.25^{\circ}-35^{\circ} \mathrm{C}\right)$ e recomendada para $A$. hypogaea $\left(20^{\circ}-30^{\circ} \mathrm{C}\right)$, todas com luz (experimento 2$)$.

\begin{tabular}{ccccc}
\hline \multirow{2}{*}{ Tratamentos } & \multicolumn{2}{c}{ Plântulas } & Mortas & Dementes \\
\cline { 2 - 5 } & Normais & Anormais & $11 \mathrm{~b}$ & $3 \mathrm{~b}$ \\
\hline $20-30^{\circ} \mathrm{C}$ & $73 \mathrm{a}$ & $13 \mathrm{a}$ & $13 \mathrm{ab}$ & $4 \mathrm{ab}$ \\
$25-30^{\circ} \mathrm{C}$ & $66 \mathrm{ab}$ & $17 \mathrm{a}$ & $15 \mathrm{a}$ & $5 \mathrm{a}$ \\
$25-35^{\circ} \mathrm{C}$ & $62 \mathrm{~b}$ & $18 \mathrm{a}$ & \\
\hline
\end{tabular}

As médias dentro de cada coluna seguidas da mesma letra não diferem entre si pelo teste de Duncan, a 5\% de probabilidade.

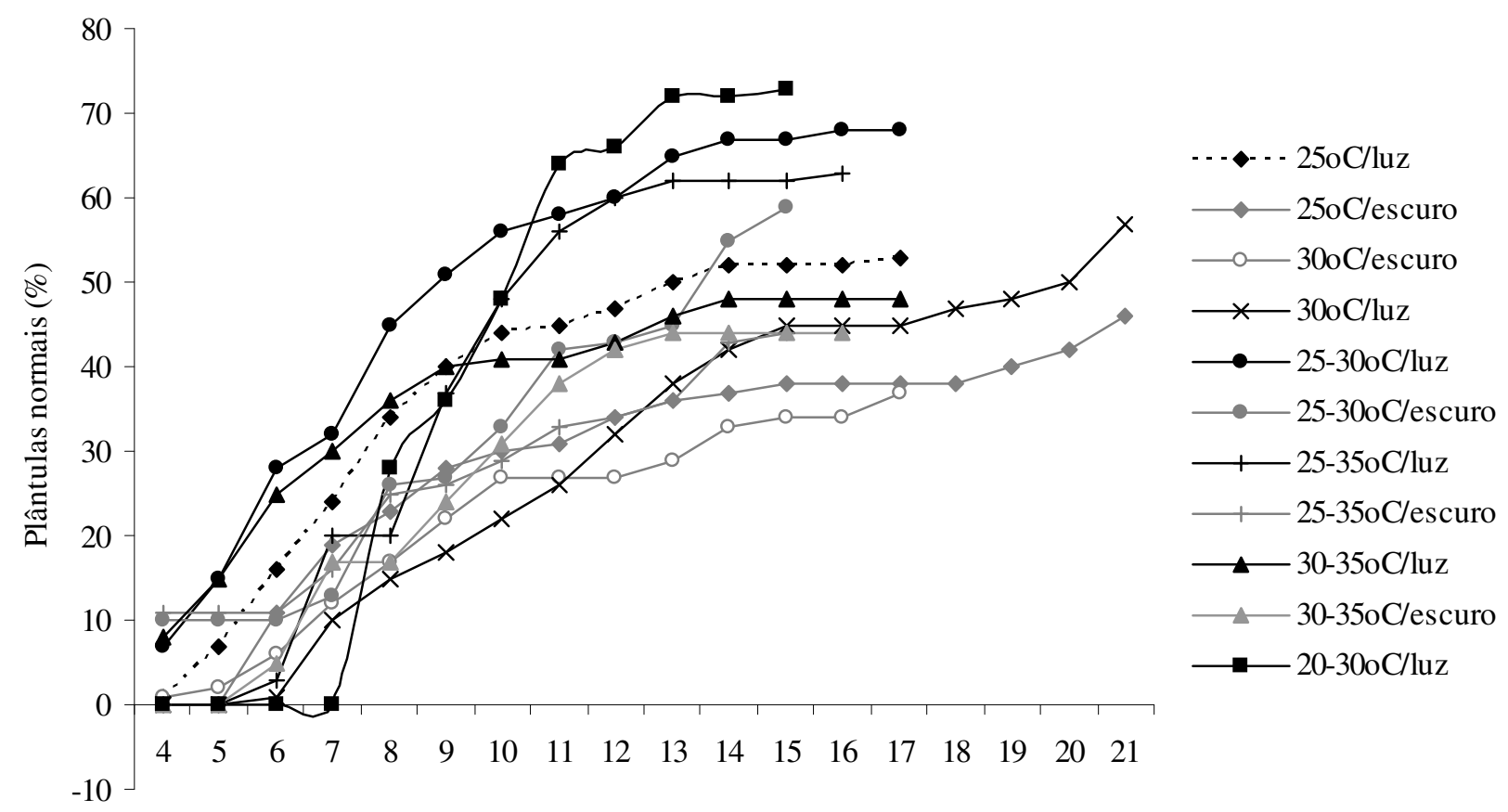

Dias

FIGURA 1. Percentagens de plântulas normais de Arachis pintoi acumulados de 4 a 21 dias após o início do teste de germinação. 
Estabeleceu-se a primeira contagem aos oito dias, já que neste período se encontrava um número suficiente de plântulas normais que pudesse ser retirado. O último dia do teste foi delimitado aos 14 dias, quando foi possível observar uma estabilidade na germinação, não ocorrendo mais o aparecimento de novas plântulas, restando apenas sementes duras e mortas (Figura 1).

\section{CONCLUSÕES}

As temperaturas alternadas de $20^{\circ}-30^{\circ} \mathrm{C}$ e $25^{\circ}-30^{\circ} \mathrm{C}$, com luz, são as mais recomendadas para a condução do teste de germinação de sementes de amendoim forrageiro, sem o uso de técnicas de superação de dormência.

A temperatura de $30^{\circ} \mathrm{C}$, com luz, promove o maior percentual de germinação entre as temperaturas constantes, mas inferior às alternadas.

A primeira contagem deve ser realizada oito dias após o início do teste e a segunda, após 14 dias.

A utilização de temperaturas alternadas promove diminuição no número de sementes duras ao final do teste de germinação, devido a superação da dormência das sementes.

\section{REFERÊNCIAS}

BEWLEY, J.D.; BLACK, M. Seeds: physiology of development and germination. 2 ed. New York: Plenum Press, 1994. p. 445.

BRASIL. Ministério da Agricultura, do Abastecimento e da Reforma Agrária. Secretaria Nacional de Defesa Agropecuária. Regras para Análise de Sementes. Brasília, DF, 1992. 365p.

CARVALHO, N.M.; Nakagawa, J. Sementes: ciência, tecnologia e produção. 4ed. Jaboticabal: FUNEP, 2000. 588p.

FERGUSON, J.E.; CARDOZO, C.I.; SÁNCHEZ, M.S. Avances y perspectivas en la producción de semillas de Arachis pintoi. Pasturas Tropicales, Cali, v.14, n.2, p.14-22, 1992.

FERGUSON, J.E. Biología de la Semilla y Sistemas de Producción de Semilla para Arachis pintoi. In: KERRIDGE, P.C. (Ed.). Biología y Agronomía de Especies Forrajeras de Arachis. Cali, Colombia: Centro Internacional de Agricultura Tropical, 1995. p.131-143.
FISHER, M.J.; CRUZ, P. Algunos Aspectos de la Ecofisiología de Arachis pintoi. In: KERRIDGE, P.C. (Ed.). Biología y Agronomía de Especies Forrajeras de Arachis. Cali, Colombia: Centro Internacional de Agricultura Tropical, 1995. p.56-75.

LABORIAU, L.G. A germinação das sementes. Washington, Organização dos Estados Americanos, 1983. 174p.

LASCANO, C.E. Valor nutritivo y producción animal del Arachis forrajero. In: KERRIDGE, P.C. (Ed.). Biología y Agronomía de Especies Forrajeras de Arachis. Cali, Colombia: Centro Internacional de Agricultura Tropical, 1995. P. 117-130.

PIZARRO, E.A.; RINCÓN, A. Experiencia Regional con Arachis Forrajero en América del Sur. In: KERRIDGE, P.C. (Ed.). Biología y Agronomía de Especies Forrajeras de Arachis. Cali, Colombia: Centro Internacional de Agricultura Tropical, 1995. p.155-169.

PONS, T.L. Seed Responses to Light. In: FENNER, M. (Ed.). Seeds: the ecology of regeneration in plant communities. Wallingford: CABI Publishing, 2000. p.237-260.

POPINIGIS, F. Fisiologia da Semente. 2 ed., Brasília: AGIPLAN. 1985.289p.

PROBERT, R.J. The Role of Temperature in the Regulation of Seed Dormancy and Germination. In: FENNER, M. (Ed.). Seeds: the ecology of regeneration in plant communities. Wallingford: CABI Publishing, 2000. p.261-292.

PROBERT, R.J.; SMITH, R.D.; BIRCH, P. Germination responses to light and alternating temperatures in European populations of Dactylis glomerata L. V. The principal components of the alternating temperature requirements. New Phytologist, v.102, p.133-142, 1986.

ROBERTS, E.H.; BENJAMIN, S.K. The interactions of light, nitrate and alternating temperatures on the germination of Chenopodium album, Capsella bursa-pastoris and Poa annua before and after chilling. Seed Science and Technology, v.7, p.379-392, 1979.

ROCHA, F.F. Análise de germinação. In: Curso sobre Análise de Sementes. Convênio UFPEL/MA/AGIPLAN, Pelotas, p.48-73. 1975.

STEINBAUER, G.P.; GRIGSBY, B. Interactions of temperatura, Light and moistening agent in the germination of leed seeds. Weeds, v.5, p.681-688, 1957.

TOOLE, E.H.; KOCH, E.J. Light and temperature controls of dormancy and germination in bentgrass seeds. Crop Science, v.17, p.806-810, 1977.

TOTTERDELL, S.; ROBERTS, E.H. Characteristics of alternating temperatures which stimulate loss of dormancy in seeds of Rumex obtusifolius L. and $R$. crispus L. Plant, Cell and Environment, v.3, p.3-12, 1980.

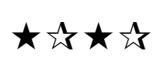

Revista Brasileira de Sementes, vol. 29, n 3, p. 61-66, 2007 